\title{
Referral patterns and proximity to palliative care inpatient services by level of socio-economic disadvantage. A national study using spatial analysis
}

David C Currow ${ }^{1 *}$, Samuel Allingham², Sonia Bird², Patsy Yates ${ }^{3}$, Joanne Lewis ${ }^{4}$, James Dawber ${ }^{2}$ and Kathy Eagar ${ }^{2}$

\begin{abstract}
Background: A range of health outcomes at a population level are related to differences in levels of social disadvantage. Understanding the impact of any such differences in palliative care is important. The aim of this study was to assess, by level of socio-economic disadvantage, referral patterns to specialist palliative care and proximity to inpatient services.
\end{abstract}

Methods: All inpatient and community palliative care services nationally were geocoded (using postcode) to one nationally standardised measure of socio-economic deprivation - Socio-Economic Index for Areas (SEIFA; 2006 census data). Referral to palliative care services and characteristics of referrals were described through data collected routinely at clinical encounters. Inpatient location was measured from each person's home postcode, and stratified by socio-economic disadvantage.

Results: This study covered July - December 2009 with data from 10,064 patients. People from the highest SEIFA group (least disadvantaged) were significantly less likely to be referred to a specialist palliative care service, likely to be referred closer to death and to have more episodes of inpatient care for longer time. Physical proximity of a person's home to inpatient care showed a gradient with increasing distance by decreasing levels of socio-economic advantage.

Conclusion: These data suggest that a simple relationship of low socioeconomic status and poor access to a referral-based specialty such as palliative care does not exist. Different patterns of referral and hence different patterns of care emerge.

Keywords: Palliative care, Socio-economic disadvantage, Service planning, Referral patterns

\section{Background}

Specialist palliative care services in Australia have grown rapidly over the last 20 years [1]. The site and model of service delivery have commonly developed in a way which is defined by local initiatives and have been ad hoc. A fundamental question arises as to whether there is evidence of discrepancies in referral to, or proximity to specialist palliative care services $[1,2]$. Are services distributed across the community in way that reflects

\footnotetext{
* Correspondence: david.currow@flinders.edu.au

${ }^{1}$ Discipline, Palliative and Supportive Services, Flinders University, 700

Goodwood Rd, Daw Park, South Australia 5041, Australia

Full list of author information is available at the end of the article
}

the populations that they serve? [3] These concerns are supported by existing evidence for limited social and geographical access to palliative care services for certain groups in Australia [4].

Internationally, inequity in palliative care service provision has been demonstrated [5,6]. Previous research has suggested that lower socio-economic status has been associated with poorer utilisation of palliative care services [7]. Inequity in the distribution of palliative care services in disadvantaged areas [5] and increased travel times to specialist palliative care facilities from low socioeconomic areas [8] have been shown to further exacerbate disparities in care for people from lower socio-

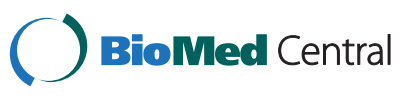


economic groups. Lower levels of palliative care service uptake by socioeconomically disadvantaged groups are considered to be driven by health and social service structures, and individual characteristics $[9,10]$.

The relationship between the social determinants of health and well-being demands a commitment to address any inequitable access to services [11]. The foundations of Australia's universal health care coverage, the established evidence for health service inequity and the vast geographical expanse of the continent confirms the need to ensure that utilisation of services is optimised for all people. To date, there are limited Australian data identifying any difference in access to palliative care services by socio-economic status.

Providing quality palliative care is not simply a social good. Emerging evidence demonstrates benefits to: patients (better symptom control, better survival, better adjustment to the disease process, better support); [12-22] caregivers (improved survival and adjustment having relinquished the role, better support in the role); [19,20,23-25] health services (fewer inpatient bed days, few admissions, lower overall costs); [21,26,27] and health practitioners (better support) [28]. These benefits then would suggest that there needs to be an assessment of referral to and proximity to services in order to ensure these benefits are available to all the people with the most complex end-of-life needs.

The use of linked population and healthcare data with geographic information (geocoding) has contributed to understanding service coverage [29-31]. The aims of this study are to examine:

i. Referral to specialist palliative care services by socialeconomic disadvantage and clinical phase [32]; and

ii. Proximity to, and utilization of inpatient services

\section{Methods}

\section{Study setting}

The Palliative Care Outcomes Collaboration (PCOC) is a national quality initiative to improve the clinical care of people with life-limiting illnesses. National coverage has grown rapidly since its inception in 2006 and, to date, more than $80 \%$ of all people seen by specialist palliative care services in Australia have data collected at point-of-care from referral until death. As a collateral opportunity, PCOC has allowed the identification of services that provide specialist palliative care nationally, including non-participating services.

Data are divided into three levels: patient data are collected once at the time of referral to the service; episode data are recorded each time the physical place of care changes; and phase data are coded with changes in clinical condition [32]. The clinical classification of 'phase' has four divisions relevant to this study: 'stable,' unstable', 'deteriorating' and 'terminal'. Independently of diagnosis, these descriptors reflect the last months of life and, in PCOC, are recorded at least weekly (for inpatient care) or with every clinical encounter (for community care) [32].

The Socio-Economic Index for Areas - Index of Disadvantage (SEIFA) based on data from the 2006 Australian Census is a summary measure of socio-economic conditions and is generated by the Australian Bureau of Statistics (ABS), and is updated after each census. The 2006 SEIFA score relates directly to the patient cohort reported here. This measure allocates a social disadvantage index to each postal area in Australia using census-derived variables including income, educational attainment, levels of employment and the number of households with a car [33]. In this study, the SEIFA index was collapsed into three SEIFA groups - low (most disadvantaged), medium and high (most advantaged) to represent three basic socio-economic levels. These three groups each represent approximately one third of the Australian population. The postcode of each patient's residence could then be allocated to one of the three SEIFA groups.

To understand service utilisation, the proportion of referrals to specialist palliative care services from each SEIFA tertile and the clinical status (phase) at the start of palliative care episodes are compared.

To understand proximity to services, postcodes with geographical locators (longitude and latitude) allow mapping of spatial relationships. In this study, those spatial relationships were measured between the patients' residences' postcodes and specialist inpatient palliative care services' postcodes if they received palliative care. Straight-line distances were calculated from a patient's residence to the specialist palliative care service, allowing an estimate of proximity to services to be developed.

\section{Analyses}

Chi-square, t-tests and analysis of variance (ANOVA) were used to compare the different measures of interest across the three SEIFA groupings as appropriate. Bonferroni corrections were applied as appropriate. A linear regression model fitted to log transformed distances was generated to adjust for the effect on SEIFA of age of the population who are dying.

Ethical oversight of this program is provided by the Human Research Ethics Committee of the University of Wollongong. This is a secondary analysis of de-identified aggregated patient and service data.

\section{Results}

Study data

The study used data from July 1 to December 31, 2009 collected at referral to specialist palliative care services across Australia. For geocoding and for socio-economic 
Table 1 Socio-Economic Index for Areas (2006 Census data; SEIFA) group for services that are and are not participating in the Australian Palliative Care Outcomes Collaboration (PCOC)

\begin{tabular}{lccc}
\hline & \multicolumn{3}{c}{ SEIFA group } \\
\cline { 2 - 4 } & Low & Medium & High \\
\hline Participating services $(n=91)$ & $47.3 \%$ & $28.6 \%$ & $24.2 \%$ \\
Non-participating services $(n=88)$ & $35.2 \%$ & $37.5 \%$ & $27.3 \%$ \\
\hline
\end{tabular}

disadvantage, patient and service level postcodes data when first recorded were used in the analyses.

\section{Study setting}

In total, 91 of the estimated 179 palliative care services that existed at this time participated in the PCOC data collection during the period. (Table 1) Fifty four percent of these services were in major cities, $32 \%$ in inner regional centres and $14 \%$ in outer regional or rural areas. While these 91 services represented $51 \%$ of palliative care services, it is estimated that they treated more than $80 \%$ of people referred to specialised palliative care services. These 91 services provided 751 (69.3\%) of the estimated 1,084 specialist palliative care inpatient beds. Services provided various combinations of inpatient, consultative and community-based services (home visits and outpatient clinic appointments).

\section{Patient population}

In this period, 10,064 patients had data collected prospectively by PCOC. These people had 12,523 episodes of care (where an 'episode' changes each time the place of care changes). Overall, $54 \%$ of referrals were males (56\% in the lowest SEIFA group; Table 2). The average age of the highest SEIFA group was significantly higher than the other two groups ( $\mathrm{p}=0.002$; Table 3 ). Eighty-two percent of those with a recorded diagnosis had cancer as their primary life-limiting illness and this proportion was the same for all three SEIFA groups (Table 2).
Table 3 Demographic and proximity data at a patient level for all people in the Australian Palliative Care Outcomes Collaboration database July 1 - December 31, 2009, by Socio-Economic Index for Areas of Disadvantage (2006 Census data; SEIFA)

\begin{tabular}{|c|c|c|c|c|}
\hline \multirow[t]{2}{*}{ Patient-level data } & & \multicolumn{3}{|c|}{ SEIFA Group } \\
\hline & & Low $^{4}$ & Medium & $\mathrm{High}^{4}$ \\
\hline \multirow[t]{3}{*}{ Age (years) } & Mean $^{2}$ & 70.3 & 70.1 & 71.3 \\
\hline & Median & 72 & 72 & 73 \\
\hline & $\mathrm{IQR}^{1}$ & 19 & 20 & 20 \\
\hline \multirow{3}{*}{$\begin{array}{l}\text { Distance from inpatient } \\
\text { care (kilometres) }\end{array}$} & Mean $^{3}$ & 43.4 & 29.9 & 14.6 \\
\hline & Median & 9.9 & 9.4 & 8.1 \\
\hline & $\mathrm{IQR}^{1}$ & 25.8 & 14.6 & 9.9 \\
\hline
\end{tabular}

1 Inter quartile range.

${ }^{2}$ High SEIFA group significantly older than both other groups (multiple t-test: largest $\mathrm{p}=0.002$ ).

${ }^{3}$ Low SEIFA group significantly greater than both other groups (multiple t-test: largest $\mathrm{p}<0.001$ ).

${ }^{4}$ SEIFA low - most disadvantaged; SEIFA high - most advantaged.

Referral to, and care provided by specialist palliative care services by socio-economic status

Using Chi-square, the distribution of referrals to specialist palliative care services was significantly lower for people from the highest SEIFA group (lowest 3744 (37.2\%), medium 3466 (34.5\%) and highest 28.3\%; $\mathrm{p}<0.001)$. This was also reflected in a significantly lower number of episodes of care provided. As a marker of timeliness of referral, people from the highest SEIFA group were also significantly less likely to be classified to the palliative care 'stable' phase $(19.4 \%$ vs $22.6 \%$ (medium) and 24.2\% (lowest); $\mathrm{p}=0.001-$ ) [31]. (Table 4) Episodes of care were significantly more likely to be in the inpatient setting for people from the highest SEIFA group (71\% compared with $63 \%$ for the other two groups; $\mathrm{p}<0.001)$ with significantly longer mean inpatient lengths of stay (highest 13.9 days; medium 11.6 days and lowest 11.5 days; $\mathrm{p}<-0.001$ ) (Table 5).

Table 2 Demographic and referral data at a patient level for all people in the Australian Palliative Care Outcomes Collaboration database July 1 - December 31, 2009, by Socio-Economic Index for Areas of Disadvantage (2006 Census data; SEIFA)

\begin{tabular}{|c|c|c|c|c|c|c|c|c|c|}
\hline \multicolumn{2}{|c|}{ Patient-level data } & \multicolumn{8}{|c|}{ SEIFA Group } \\
\hline & & \multicolumn{2}{|l|}{ Low $^{2}$} & \multicolumn{2}{|c|}{ Medium } & \multicolumn{2}{|c|}{$\mathrm{High}^{2}$} & \multicolumn{2}{|l|}{ Total } \\
\hline & & $\mathrm{n}$ & $\%$ & $\mathrm{n}$ & $\%$ & $\mathrm{n}$ & $\%$ & $\bar{n}$ & $\%$ \\
\hline \multirow[t]{2}{*}{ Diagnosis } & Cancer & 2,781 & $83 \%$ & 2,580 & $81 \%$ & 1,961 & $82 \%$ & 7,322 & $82 \%$ \\
\hline & Non cancer & 577 & $17 \%$ & 614 & $19 \%$ & 437 & $18 \%$ & 1,628 & $18 \%$ \\
\hline \multirow[t]{2}{*}{ Gender } & Male & 2,114 & $56 \%$ & 1,869 & $54 \%$ & 1,447 & $51 \%$ & 5,430 & $54 \%$ \\
\hline & Female & 1,630 & $44 \%$ & 1,597 & $46 \%$ & 1,396 & $49 \%$ & 4,623 & $46 \%$ \\
\hline \multicolumn{2}{|c|}{ Referral to specialist palliative care service ${ }^{1}$} & 3,751 & $37 \%$ & 3,470 & $34 \%$ & 2,843 & $28 \%$ & 10,064 & $100 \%$ \\
\hline
\end{tabular}

${ }^{1}$ Significant difference in SEIFA groups (Chi square: $\left.p<0.001\right)$.

2 SEIFA low - most disadvantaged; SEIFA high - most advantaged. 
Table 4 Episode of care level data in the Australian Palliative Care Outcomes Collaboration database July 1 - December 31, 2009, by Socio-Economic Index for Areas of Disadvantage (2006 Census data; SEIFA)

\begin{tabular}{|c|c|c|c|c|c|c|c|c|c|}
\hline \multirow[t]{3}{*}{ Episode-level data } & & \multicolumn{8}{|c|}{ SEIFA group } \\
\hline & & \multicolumn{2}{|c|}{ Low $^{3}$} & \multicolumn{2}{|c|}{ Medium } & \multicolumn{2}{|c|}{$\mathrm{High}^{3}$} & \multicolumn{2}{|c|}{ Total } \\
\hline & & $\mathrm{n}$ & $\%$ & $\mathbf{n}$ & $\%$ & $\mathrm{n}$ & $\%$ & $\mathrm{n}$ & $\%$ \\
\hline \multirow[t]{2}{*}{ Place of care ${ }^{1}$} & Inpatient & 2,919 & $63 \%$ & 2,871 & $63 \%$ & 2,358 & $71 \%$ & 8,148 & $65 \%$ \\
\hline & Community & 1,703 & $37 \%$ & 1,693 & $37 \%$ & 979 & $29 \%$ & 4,375 & $35 \%$ \\
\hline \multirow{4}{*}{$\begin{array}{l}\text { Phase at beginning of a patient's } \\
\text { palliative care episode }\end{array}$} & Stable ${ }^{2}$ & 1,076 & $24 \%$ & 971 & $23 \%$ & 620 & $19 \%$ & 2,667 & $22 \%$ \\
\hline & Unstable & 1,786 & $40 \%$ & 1,639 & $38 \%$ & 1,405 & $44 \%$ & 4,830 & $40 \%$ \\
\hline & Deteriorating & 1,246 & $28 \%$ & 1,367 & $32 \%$ & 960 & $30 \%$ & 3,573 & $30 \%$ \\
\hline & Terminal & 343 & $8 \%$ & 320 & $7 \%$ & 219 & $7 \%$ & 882 & $7 \%$ \\
\hline
\end{tabular}

(Episode changes every time physical place of care changes).

1 High SEIFA group significantly different from both other groups (multiple chi-square: largest $\mathrm{p}<0.001$ ).

${ }^{2}$ High SEIFA group has a significantly lower proportion of episodes starting 'Stable'(multiple chi-square; largest $\mathrm{p}=0.001$ ).

${ }^{3}$ SEIFA low - most disadvantaged; SEIFA high - most advantaged.

Proximity to inpatient services by socio-economic status Using geocoding, for people who had at least one inpatient episode of care, there were significant differences between the proximity to that care by SEIFA. For the lowest group, distances were a mean of $43.4 \mathrm{~km}$ compared to medium $(29.9 \mathrm{~km})$ and highest $(14.6 \mathrm{~km})$ (Table 3). Using a regression model with distance as the dependent variable, and controlling for SEIFA, rurality and age, the model demonstrated that older people were significantly more likely to be proximate to inpatient services $\left(R^{2} 0.78\right.$; for age, $\left.p<0.001\right)$.

\section{Discussion}

This study confirms that there are significant differences in the uptake of, and proximity to specialist palliative care services in Australia. These differences are associated with socio-economic status in a complex way. For

Table 5 Episode of care level data in the Australian Palliative Care Outcomes Collaboration database July 1 - December 31, 2009, by Socio-Economic Index for Areas of Disadvantage (2006 Census data; SEIFA)

\begin{tabular}{cccccc}
\hline & & \multicolumn{3}{c}{ SEIFA group } \\
\cline { 3 - 5 } Episode-level data & & Low $^{\mathbf{3}}$ & Medium & High $^{\mathbf{3}}$ \\
\hline $\begin{array}{c}\text { Episode length } \\
\text { (elapsed days) }\end{array}$ & $\begin{array}{c}\text { Inpatient care } \\
\text { (excluding day } \\
\text { only admissions) }\end{array}$ & Mean & 11.5 & 11.6 & 13.9 \\
& IQR & 12 & 12 & 14 \\
& $\mathrm{n}$ & 2,802 & 2,768 & 2,257 \\
& $\begin{array}{c}\text { Community care } \\
\text { (including day }\end{array}$ & Mean & 46.9 & 42.9 & 41.8 \\
only admissions) & Median & 2 & 19 & 21 \\
& IQR & 57 & 49 & 44
\end{tabular}

(Episode changes every time physical place of care changes).

${ }^{1}$ High SEIFA group significantly different from both other groups (multiple t-test: largest $\mathrm{p}<0.001)$.

${ }^{2}$ High SEIFA group significantly different from both other groups (multiple t-test:

${ }^{3}$ SEIFA low - most disadvantaged; SEIFA high - most advantaged. the highest SEIFA category (those who were least disadvantaged) there was: lower uptake of specialist palliative care services; later in the course of the illness; with referral less likely to be in the stable phase; better proximity to inpatient services; and longer duration of inpatient care [34,35]. These data support previous populationbased studies in the United States, Canada and Australia that observed disparities in access to specialist palliative care services by socio-economic disadvantage, although the patterns appear somewhat different to previous reports [36-38].

Not all studies on disparities on referral to specialist palliative care services have seen socio-economic differences [39]. Higher levels of community-based care in the lowest tertile shifts the responsibility of care to families and friends, including the financial cost of caring. Such differences in community-based care are amplified if the most advantaged SEIFA group are able to access a range of services including user-pays nursing and home care $[34,40]$.

Proximity to specialist palliative care services may have significant implications for health service delivery more broadly. In an analysis of more than 28,000 people over the age of 65 who died of cancer within one year of diagnosis, while controlling for key demographic and clinical factors, physical proximity to hospice services was an independent predictor of less aggressive end-of-life care [41].

\section{Needs based services}

Do people from lower socio-economic strata have different needs? There is no evidence that needs in the most disadvantaged communities are any less and, arguably, given the social deprivation experienced across the life span, needs will be greater [42]. This challenges directly hospice and palliative services to consider how to provide needs-based care that genuinely serves the whole population in order to achieve equitable outcomes (not simply 
equitable access) [34]. The patterns demonstrating higher levels of chronic illness and of earlier mortality for people from greater socio-economic disadvantage would suggest that there is an even greater focus required to ensure equitable outcomes at the end of life given, for example, the higher likelihood of dependent children given the age at which people are dying.

\section{Limitations}

These data also assume that for each SEIFA group the likelihood of death is equal. The discrepancy in access is likely to be greater given that socio-economic status at the end of life is going to be lower for a significant proportion of the population given diminishing disposable income for older people. The services not represented in the patient-level data were more likely to be rural where greater socio-economic disadvantage is also likely. Discrepancies in the way services are utilised are magnified in subtle ways - the costs of community-based care are borne largely by the family and the proportion of care provided in the community is greatest in the most disadvantaged group in the community. Likewise, the costs of travel to inpatient units will be disproportionately borne by those least able to afford it.

\section{Limitations of the study (sample)}

The study does not include all services in Australia, but the missing services tend to be smaller more rural services where poor proximity to care will add to disadvantage. Their inclusion would likely magnify the effects seen in the data presented. Coding has been done for the services that are missing, but the populations who access these services are not available in this data set.

People who already experience geographic isolation from services may simply never be referred and hence not represented in this sample further underestimating disparities. Only people who used designated inpatient palliative care beds form the basis of this analysis. This therefore underestimates the impact of distance for people who chose not to have inpatient care because the distances were too great, or had care at a hospital closer to home in a non-designated bed.

\section{Limitations of the study (data)}

The current studies refer only to distance not ease of travel which incorporates distance, mode of travel, costs and other factors that contribute to accessibility. Although absolute distances may be much shorter in metropolitan areas, travel time especially when relying on public transport may still be very challenging [43].

Using straight-line distances between the patient's postcode and the postcode of the inpatient facility, it is likely that the travel distance is underestimated for patients living in regional and rural areas. This is because postal areas are not uniform in geographic size. Hence, patients living in the same postcode as a palliative care service in a regional area (assigned a travel distance of 0 ) may need to travel much further than a patient living in a major city who lives in the same postcode as a palliative care service. While straight-line distance travelled is not an exact measure of travel burden, it acts as a strong indicative measure of access.

\section{Generalisability}

These data refer to the Australian health and social systems, thus limiting generalisability to other settings. Further, there are differential rates of coverage for the services represented: $51 \%$ of all services; $69 \%$ of all designated inpatient beds; and approximately $80 \%$ of all patients referred to specialist palliative care services in the country. As noted in Limitations, this therefore probably underestimates the differences seen for the most socially disadvantaged rural patients.

\section{Future research directions}

Research in the future should look specifically at needs, complexity of care and outcomes at the end of life by socio-economic status. Given that one in two people with an expected death will not access palliative care in Australia, population level studies are required to ensure that service delivery better incorporates needs-based access to specialist palliative care services across the community [44]. Accessibility to services needs to be geocoded for all predictable deaths accepting that only a sub-set would derive benefit from referral to specialised palliative care services.

\section{Conclusion}

Most palliative care services have had little jurisdictional or local planning. With few exceptions, this 'organic' process has been built around community interests and goodwill as well as the availability of existing hospital stock rather than sound principals of health service planning. Population-based service planning may require further affirmative action to overcome some of the geographical difficulties highlighted by this current study.

Better use of new models of care with emerging technologies such as video-conferencing for clinical consultations and family conferences may relieve some burden related to poor proximity to services. This becomes an overwhelming imperative for areas where long distances need to be travelled by caregivers, families and friends in metropolitan peri-urban and rural areas when inpatient services are dislocated from that person's own environment. 
Inpatient care needs to be accessible since much of it is required at short notice due to unexpected changes in condition. Inpatient care also needs to be physically proximate so that family and friends can continue to support patients.

\section{Competing interests}

The author(s) declare that they have no competing interests.

\section{Authors' contributions}

Conception and design DC,KE; Planning DC,KE,PY; data acquisition DC,PY,JL, KE; analysis SA,SB,JD; interpretation KE,PY,JL,DC; Drafting DC, Critical Revision DC,SA,SB,PY,JL,JD,KE. All authors read and approval the final manuscript.

\section{Acknowledgements}

Thanks to A/Prof Meera Agar for her helpful critique of the manuscript and Ms Debbie Marriott for her excellent work in preparing the manuscript for submission.

\section{Funding}

The Australian Palliative Care Outcomes Collaboration is funded by the Australian Government's Department of Health and Ageing.

\section{Author details}

${ }^{1}$ Discipline, Palliative and Supportive Services, Flinders University, 700 Goodwood Rd, Daw Park, South Australia 5041, Australia. ${ }^{2}$ Centre for Health Service Development, University of Wollongong, Wollongong, Australia. ${ }^{3}$ Faculty of Health Sciences, Queensland University of Technology, Herston, Brisbane, Australia. ${ }^{4}$ University of Technology, Broadway, Sydney, New South Wales, Australia.

Received: 4 November 2011 Accepted: 22 October 2012 Published: 23 November 2012

\section{References}

1. National Health and Hospitals Reform Commission: End of life care is everyone's affair-tackling the challenge of 'end of life'. Palliative Care Australia submission to the National Health and Hospitals Reform Commission. Canberra: Palliative Care Australia; 2008. http://www.palliativecare.org.au/ Portals/46/PCA\%20submission\%20to\%20NHHRC.pdf

2. Currow DC, Abernethy AP, Fazekas BS: Specialist palliative care needs of whole populations: a feasibility study using a novel approach. Palliat Med 2004, 18(3):239-247.

3. Allen F: EoL - Towards quality care at the end of life. Volume 1(1) Winter. Canberra: Palliative Care Australia; 2009. http://www.palliativecare.org.au/ Portals/46/EoL\%20-20Winter\%202009.pdf.

4. Rosenwax LK, McNamara BA: Who receives specialist palliative care in Western Australia-and who misses out. Palliat Med 2006, 20(4):439-445.

5. Higginson IJ, Finlay IG, Goodwin DM, Hood K, Edwards AGK, Cook A, Douglas $H$, Normand $C E$ : Is there evidence that palliative care teams alter end-of-life experiences of patients and their caregivers? J Pain Symptom Manage 2003, 25(2):150-168.

6. Decker $\mathrm{SL}$, Higginson IJ: A tale of two cities: factors affecting place of cancer death in London and New York. Eur J Public Health 2007, 17(3):285-290.

7. Beccaro M, Constantini M, Merlo DF, ESDOC Study Group: Inequity in the provision of and access to palliative care for cancer patients. Results from the Italian survey of the dying of cancer (ISDOC). BMC Publ Health 2007, 7:66

8. Wood DJ, Clark D, Gatrell AC: Equity of access to adult hospice inpatient care within north-west England. Palliat Med 2004, 18(6):543-549.

9. Hanratty B, Jacoby A, Whitehead M: Socioeconomic differences in service use, payment and receipt of illness-related benefits in the last year of life: findings from the British household panel survey. Palliat Med 2008, 22(3):248-255

10. Cardenas-Turanzas M, Grimes RM, Bruera E, Quill B, Tortolero-Luna G Clinical, sociodemographic, and local system factors associated with a hospital death among cancer patients. Support Care Cancer 2006, 14(1):71-77.
11. Mackenbach JP, Stiribu I, Roskam AJ, Schaap MM, Menvielle G, Leinsalu M, Kunst AE, European Union Working Group on Socioeconomic Inequalities in Health: Socioeconomic inequalities in health in 22 European countries. N Eng J Med 2008, 358(23):2468-2481

12. Temel JS, Greer JA, Muzikansky A, Gallagher ER, Admane S, Jackson VA, Dahlin CM, Blinderman CD, Jacobsen J, Pirl WF, Billings JA, Lynch TJ: Early palliative care for patients with metastatic non-small-cell lung cancer. N Engl J Med 2010, 363(8):733-742.

13. Bakitas M, Lyons KD, Hegel MT, Balan S, Brokaw FC, Seville J, Hull JG, Li Z, Tosteson TD, Byock IR, Ahles TA: Effects of a palliative care intervention on clinical outcomes in patients with advanced cancer: the Project ENABLE II randomized controlled trial. JAMA 2009, 302(7):741-749.

14. Miller SC, Mor V, Wu N, Gozalo P, Lapane K: Does receipt of hospice care in nursing homes improve the management of pain at the end of life? J Am Geriatr Soc 2002, 50:507-515.

15. Miller SC, Mor $V$, Teno J: Hospice enrolment and pain assessment and management in nursing homes. J Pain Symptom Manage 2003, 26(3):791-799

16. Hillier JB, Williams A, Oldham J: Hospital based palliative care teams improve the symptoms of cancer patients. Palliat Med 2003, 17(6):498-502.

17. Currow DC, Ward AM, Plummer JL, Bruera E, Abernethy AP: Comfort in the last 2 weeks of life: relationship to accessing palliative care services. Support Care Cancer 2008, 16(11):1255-1263.

18. Wallston KA, Burger C, Smith RA, Baugher RJ: Comparing the quality of death for hospice and non-hospice cancer patients. Medical Care 1988 177-182.

19. Kane RL, Bernstein L, Wales J, Leibowitz A, Stevan Kaplan S: A randomized controlled trial of hospice care. Lancet 1984, 323(8382):890-894.

20. Hughes SL, Cummings J, Weaver F, Manheim L, Braun B, Conrad K: A randomized trial of the cost effectiveness of VA hospital-based home care for the terminally ill. Health Serv Res 1992, 26(6):801-817.

21. Brumley RD, Euguidanos S, Cherin DA: Effectiveness of a home-based palliative care program for end-of-life. J Palliat Med 2003, 6(5):715-724.

22. Higginson IJ, Costantini M: Dying with cancer, living well with advanced cancer. Eur J Cancer 2008, 44(10):1414-1424.

23. Teno JM, Clarridge BR, Casey V, Welch LC, Wetle T, Sheild R, Mor V: Family perspectives on end-of-life care at the last place of care. JAMA 2004, 291(1):88-93.

24. Christakis NA, Iwashyna TL: The health impact of health care on families: a match cohort study of hospice use by descendents and mortality outcomes in surviving, widowed spouses. Soc Sci Med 2003 57(3):465-475

25. Abernethy AP, Currow DC, Fazekas BS, Luszcz MA, Wheeler JL, Kuchibhatla M: Palliative care services make a difference to short- and long-term caregiver outcomes. Support Care Cancer 2008, 16(6):585-597.

26. Constantini M, Higginson IJ, Bon L, Orengo MA, Garrone E, Henriquet F, Bruzzi P: Effect of palliative home care team on hospital admissions among patients with advanced cancer. Palliat Med 2003, 17(4):315-321.

27. Connor SR, Pyenson B, Fitch K, Spence C, Iwasaki K: Comparing hospice and non-hospice patient survival among patients who die within a three-year window. J Pain Symptom Manage 2007, 33(3):238-246.

28. Muir JC, Daly F, Davis MS, Weinberg R, Heintz JS, Paivanas TA, Beveridge R: Integrating palliative care into the outpatient, private practice oncology setting. J Pain Symptom Manage 2010, 40(1):126-135.

29. Hanratty B, Goldacre M, Griffith M, Whitehead M, Capewell S: Making the most of routine data in palliative care research-a case study analysis of linked hospital and mortality data on cancer and heart failure patients in Scotland and Oxford. Palliat Med 2008, 22(6):744-749.

30. Cinnamon J, Schuurman N, Crooks VA: A method to determine spatial access to specialized palliative care services using GIS. BMC Health Serv Res 2008, 8:140.

31. Crooks VA, Castleden H, Schuurman N, Hanlon N: Visioning for secondary palliative care service hubs in rural communities: a qualitative case study from British Columbia's interior. BMC Palliat Care. 2009, 8:15.

32. Eagar K, Green J, Gordon R: An Australian casemix classification for palliative care: technical development and results. Palliat Med 2004, 18(3):217-226.

33. Anon: Australian bureau of statistics (2008) census of population and housing: socio-economic indexes for areas (SEIFA), Australia - data only, 2006, cat. no. 
2033.0.55.001, ABS. Canberra. http://www.abs.gov.au/ausstats/abs@.nsf/mf/ 2033.0.55.001 Canberra, Australia. Accessed 11 ${ }^{\text {th }}$ October, 2011.

34. Walshe C, Todd C, Caress A, Chew-Graham C: Patterns of access to community palliative care services: a literature review. J Pain Symptom Manage 2009, 37(5):884-912.

35. Sims A, Radford J, Doran K, Page H: Social class variation in place of cancer death. Palliat Med 1997, 11(5):369-373.

36. O'Mahony S, McHenry J, Snow D, Cassin C, Schumacher D, Selwyn PA: A review of barriers to utilization of the medicare hospice benefits in urban populations and strategies for enhanced access. J Urban Health 2008, 85(2):281-290.

37. Burge $\mathrm{Fl}$, Lawson B, Johnston G: Home visits by family physicians during the end-of-life: Does patient income or residence play a role? BMC Palliat Care 2005, 4(1):1.

38. Currow DC, Agar M, Sanderson C, Abernethy A: Populations who die without specialist palliative care: Does lower uptake equate with unmet need? Palliat Med 2008, 22(1):43-50

39. Gray JD, Forster DP: Factors associated with utilization of specialist palliative care services: a population based study. J Public Health Med 1997, 19(4):464-469.

40. Silviera MJ, Kabeto MU, Langa KM: Net worth predicts symptom burden at the end of life. J Palliat Med 2005, 8(4):827-837.

41. Earle CC, Neville BA, Landrum M, Ayanian JZ, Block SD, Weeks JC: Trends in the aggressiveness of cancer care near the End of life. J Clin Oncol 2004, 22(2):315-321

42. Clark CR: Social deprivation increases workload in palliative care of terminally ill patients. Br Med J 1997, 314(7088):1202.

43. Kirkpatrick SI, Tarasuk V: Assessing the relevance of neighbourhood characteristics to the household food security of low-income Toronto families. Public Health Nutr 2010, 13(7):1139-1148.

44. Waller A, Girgis A, Currow D, Lecathelinais C: Development of a palliative care needs assessment tool (PC-NAT) for use by multi-disciplinary health professionals. Palliat Med 2008, 22(9):956-964.

doi:10.1186/1472-6963-12-424

Cite this article as: Currow et al:: Referral patterns and proximity to palliative care inpatient services by level of socio-economic

disadvantage. A national study using spatial analysis. BMC Health Services Research 2012 12:424.

\section{Submit your next manuscript to BioMed Central and take full advantage of:}

- Convenient online submission

- Thorough peer review

- No space constraints or color figure charges

- Immediate publication on acceptance

- Inclusion in PubMed, CAS, Scopus and Google Scholar

- Research which is freely available for redistribution 